had a chance and takes a great interest in astronomy, for instance, and goes up and watches the professor in the observatory, he would gather a great many ideas. Give him a chance at the observatory. That is what we want. It is a bold idea, I know, but that is what we want. If there is danger of their breaking it, they can be watched the more carefully. I often think if I could take two or three boys and bring them up and attend to their education and pay no attention to anything else whatever, I could fix them in ten year's time so that they could earn so much more than I can, that it would pay in the long run to apply one's time and attention to it. [Laughter.]

Professor Jackson:- Mr. President, Mr. Owens states that he does not care to reply. I have no reply to make, except to thank the various gentlemen who have taken part in the discussion. Their recognition of the advantages of an engineering education is very gratifying.

I wish to add a remark to Lieutenant Sprague's definition of an electrical engineer, that he should be one-tenth mechanical, one-tenth electrical and the rest he didn't know what. Dr. Nichols says the rest should be man. I want to add to that, what is left should be good, manly common sense. [Applause.]

I want to especially thank Mr. Lockwood for his remarks, which were evidently very pleasing to us all, and I want to assure Mr. Lockwood that we consider one of the necessities of an electrical engineering course is to teach the students how to read technical journals without wasting too much time, and also how to make references to current books of the day which it is impossible to thoroughly read but which must be used more or less.

I also wish to thank Mr. Upton for his recognition of the necessity of practical instruction in engineering education. I feel that the practical is demanded at the present day. The theoretical side has been developed. I do not think it is necessary for an electrical engineer to be able to read Maxwell through all its parts. I would scarcely claim that distinction myself. In fact, there are very few pages in Maxwell that would be enjoyed as reading matter.

As to the question of shop apprentices, I think it is well, under ceriain circumstances, for a student to enter a properly arranged apprentice course in a first-class commercial shop. The size of the shop may be of little importance, but if it is not first-class the student will probably be wasting his time.

Finally, I want to say a word about our universities. If a man comes to a university with the view of learning something special, and doing good work, and making use of what he can learn, he will receive every attention and fully as much assistance as any man who takes the regular prescribed course.

The following paper on "A New Rheostat" was then read by the author. 


\section{A NEW RHEOSTAT.}

BY CHARLES E. CARPENTER.

It is a well-known fact that an insulated wire suspended in the air, will carry a given amount of current at a lower temperature than the same wire without this covering of insulation under the same conditions. This is obviously due to the fact that while the cross-section of both conductors is the same, the one which is insulated has a much larger radiating surface than the bare wire, and therefore dissipates the heat conducted through the insulation more rapidly. Suppose, for convenience of illustration, we conceive a conductor to be first covered with some insulating sub-

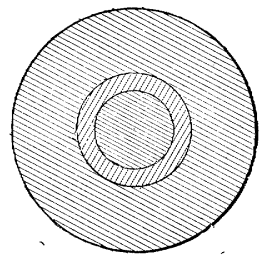

FrG. 1.

stance, and then enclosed within a metallic tube so that its insulated surface shall fit perfectly within and against the inside surface of the tube, as shown in section in Fig. 1. We thus have a comparatively small conductor with a largely increased radiating surface, and the insulation between the two metallic bodies does not materially obstruct the flux of heat from the conductor to the outside metallic shell.

One of the early experiments of the writer in electric heating -in fact, his first successful apparatus-consisted of two iron plates, 
between which was interposed an iron resistance wire of the "reflex" or "zig zag" type insulated from both plates by means of thin sheets of mica or asbestos paper, with screws passing through one plate and engaging the other. By this means the plates could be pressed into close relation to the resistance wire. By connecting this apparatus to a constant potential system, the current at first would be comparatively large, but would gradually decrease as the temperature of the plates increased until a point was reached where the heat was dissipated as fast as generated. By tightening or loosening the screws, the current could then be either increased or decreased. This increase of the current by increasing the mechanical pressure is due to a reduction of temperature effected by the more intimate heat conducting relations between the resistance and plate, facilitating the flux of heat.

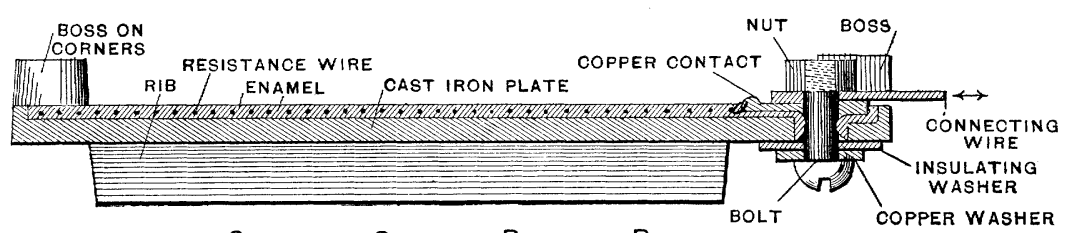

Section of Standard Rheostat Plate

FIG. 2.

The better the conduction for heat is made, the less will be the difference of temperature between the wire and the outside radiating surface plate, and it follows that the larger the surface of the radiating plates which are closely impressed upon the conductor, the larger will be the current-carrying capacity of the latter.

These experiments are cited as an illustration leading to a thorough appreciation of the extent to which the current capacity of a resistance wire may be carried, as it is upon this principle, but with modifications of construction, that the new and improved rheostat herein described is based, that is, a resistance wire of small cross-section is used, but a large radiating capacity is secured by placing the wire in such relations to a metal plate as to practically increase its radiating surface to a very great extent.

The results of the experiments described above suggested a modified form of construction, one in which the resistance would be entirely covered by a medium which would protect the wire from oxidation and yet act as a good insulator of electricity and a good 
conductor of heat, and which will permanently attach the wire to, but insulate it from, a radiating plate. A special enamel was finally found by the writer to be suitable for this purpose. A section of this construction is shown in Fig. 2.

The injurious effect of linear expansion of the resistance wire, due to heat, is avoided in this method by the " reflexed" or "zigzag" form in which the wire is bent, as shown in Fig. 3, and it has been demonstrated by experience that this linear expansion is thereby so distributed as not to injure or crack off the enamel in practice.

On this principle, since we are able to greatly reduce the crosssection of the conductor, it follows that its length is also proportionately shortened for any required resistance, and no consideration of mechanical strength of the wire enters into this construction, since it is so perfectly confined and supported on all sides.

Practice has demonstrated that 20 watts of electrical energy may be dissipated continuously to each square inch of resistance surface, without injury to the apparatus. However, on account of the excessive temperature of the plate used in close proximity to the other materials, it is not practicable to dissipate over 8 or 10 watts to the square inch in continuous service.

By placing one surface of the plate in contact with water, so that its heat is dissipated in this manner, we are able to continuously dissipate about 25 watts to the square inch. From this it will be seen that by means of water we are able to increase the capacity of a certain plate to about three times that of the same plate dissipating its heat directly into the atmosphere, or we may decrease the size of the plate to one-third for the same capacity where water is used.

In many applications, especially in railway work, apparatus is often subjected to a load far beyond its rated capacity. Rheostat plates for such service are made with narrow ribs, between which the resistance wire and enamel are placed. It is found that by means of these ribs, the safe carrying capacity of a wire is greatly increased, while the ribs serve also to protect the insulation and wires from mechanical injury.

In adapting this construction to meet practical requirements in its several applications, it is necessary to alter only in detail the general construction and distribution before explained, and shown in Fig. 3, which shows the usual plan of distributing the wires in a simple rheostat plate. Connection to the resistance wire is made 
by means of a sheet copper contact strip, one portion of which is folded back upon itself, and after inserting a portion of the wire this sheet copper is crushed about it. The joint so formed can not oxidize, since it is completely sealed by the enamel fused about it.

To divide the resistance so formed into steps, it is only necessary to place contacts for connection to the resistance wire at convenient points where the loops approach the edge of the plate, as at $a$, and so on, in Fig. 3. This particular way of locating the connecting points on one side of the plate is especially convenient in using these plates with small motors, where only few steps or divisions of the resistance are required. A modification of this

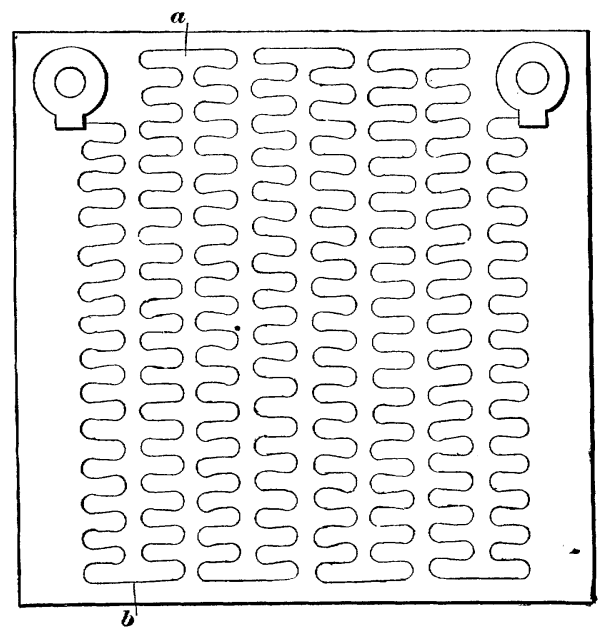

Fig. 3.

arrangement is to have the connecting points alternate from one side of the plate to the other, as at $b, a$, etc., Fig. 3. A distribution best adapted for stage or field-controlling rheostat, or one in which a great number of steps are required, is secured by arranging the resistance in a similar manner around the entire outer portion of a disk with contact connections near the circumference. Another arrangement is required in a plate of low resistance and high ampere capacity. In this case, several coils are placed in parallel and connected to a common contact piece.

The plates, with their coils and contacts distributed as above described, are well suited for use in connection with rheostat 
switches. They are provided with ribs or blades for extending their radiating surface, and lugs at the corners for convenience in attaching them to the back of the switch. Each contact of the resistance plate is connected by a very short wire or strip, to the corresponding contact piece on the rheostat switch.

A very simple and complete rheostat is made by placing the switch contacts and lever, properly insulated directly upon the radiating plate, on the surface opposite the resistance face. With this arrangement as shown in Fig. 4, a rheostat is obtained which is no larger than the switch parts alone of existing rheostats.

To make a single plate large enough to dissipate the energy

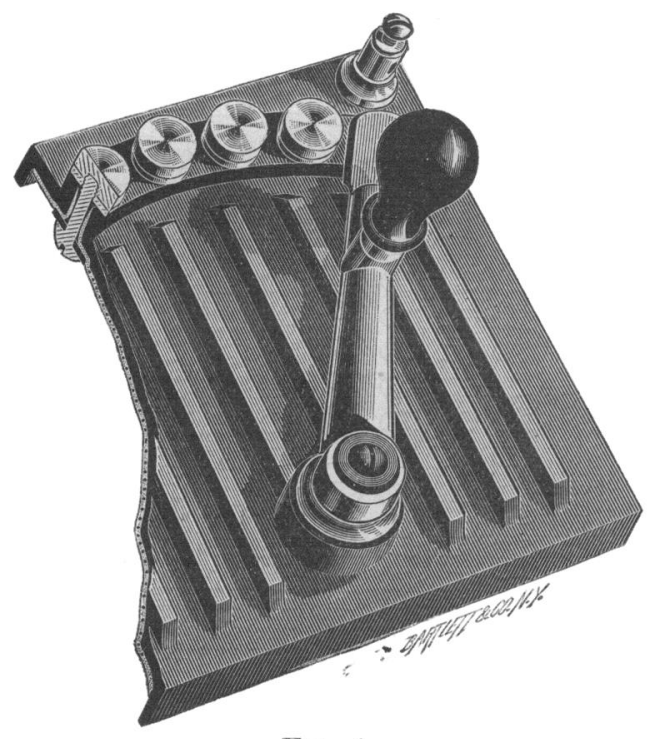

Fig. 4.

wasted in the rheostat in starting a large motor, would make it necessary to have a surface so large as to be objectionable. To avoid this difficulty, a number of plates of, say, 10 inches square, are placed in parallel with each other, but separated by sufficient space to admit of proper ventilation, and connection made from the switch contacts to the contacts upon the various plates as required With this arrangement, a rheostat of very large watt capacity may be placed within a small cubic space.

This form of rheostat, by reason of its compactness, is well adapted for use with are lamps on constant potential circuits. In 
this application, the resistance plate may be attached directly to the outside of the casing, leaving just sufficient space bet ween the casing and the plate for ventilation. Where the casing is cylindrical, the plate may be disk-shaped and placed on top of the cylindrical casing, with holes in the plate for the hooks and chimney to pass through. A valuable feature of this rheostat in this application is that it will endure exposure to the weather while in use. As an example of this, the writer knows of a case where one of these plates has now been in use on a pair of lamps for over seven months, where the plate is in a horizontal position, with the enameled side up and water dripping upon it continually.

In some places the heating effects of rheostats should be avoided. For example, in stage controllers, 5 to 10 kilowatts continuously converted into heat is extremely objectionable in warm weather. This may be remedied by conveying the heat away through the agency of running water. To accomplish this the resistance plate may be hollow, with inlet and outlet pipes connected directly to a source of water supply. By this means and with proper circulation, the plates will always be comparatively cold, and no sensible amount of heat will be given off to the surrounding atmosphere, while the size of the apparatus is so reduced as to occupy no considerable amount of space.

It is evident that numerous modifications of this general design may easily be made to adapt the apparatus to the requirements of practice.

The following facts are true regarding this form of rheostat:

1st. It is fireproof, as no combustible material enters into its construction, and it can never be submitted in normal use to a temperature so high as that towhich it is subjected in the process of manufacture.

$2 d$. It is durable, since the resistance wires cannot deteriorate from oxidation, electrolysis, etc.

$3 \mathrm{~d}$. It is compact, as it requires less than 5 per cent. of the cubic space required by the ordinary coiled wire rheostat of the same capacity.

4th. It is simple, the three necessary elements, resistance wire, insulation and radiating surface plate, being fused into one integral mass.

5 th. It is cheap, for a certain resistance and carrying capacity can be obtained by the use of a wire whose cross-section and 
length will both be but one-tenth of that required for a wire used in the air. That is, the total weight of wire, and hence the cost of the same, will be but one per cent. of that usually required.

6th. It is unaffected by heat, cold, acids, alkalies, oils and other chemicals, and for this reason is especially adapted for use in mines, breweries, marine vessels, etc., where chemical action rapidly destroys ordinary insulators. It is even practicable to operate these plates submerged in flowing water, in case it is desirable to carry off the heat in this way.

Another and important application of this method of converting electric energy into heat in a compact form is for the operation of various heating devices.

The requirements of a commercially successful electric heating device are :

1st. The concentration upon the working surface of the apparatus, of practically all of the heat produced in the resistance, thus preventing as far as possible the dissipation of heat by radiation or conduction to other bodies or parts than those it is required to heat.

$2 d$. The ability to rapidly supply a large amount of heat energy from the resistance to the working surface, through a medium having a high conductivity for heat, thus keeping the working surface at the proper temperature even when a large amount of heat is rapidly drawn from it.

$3 \mathrm{~d}$. The protection of the resistance wire from deterioration by chemical and electrical action, so that its life shall be indefinitely prolonged.

All the above requirements are fulfilled in this device.

DISCUSSION.

The President :-Gentlemen, you have heard Mr. Carpenter's interesting paper. I am sure those who have had anything to do with rheostats will appreciate the very practical and simple form in which this rheostat is presented. I see many applications of it which are of interest, and may be made practical. If there is any discussion on this paper, we shall be glad to hear it.

Mr. Sperry :-Mr. President, I would like to call out some points with reference to it. I would like to know if the rheostat has been designed for heavy currents, and if so what is the heaviest that has been used?

Mr. CarPenter:-We are manufacturing resistance plates of low resistance and high ampere capacity. In these low resistance plates, several wires are in parallel or multiple, and have a 
capacity depending upon the kind and size of the wire, from 25 to 200 amperes or more. A 50 kilowatt 500 volt motor-starter is made up of a number of these low resistance plates connected in series. Each plate has 4 wires in parallel, and each plate forms a step or division of the rheostat. The plates for this size of motor are 18 inches long and 3 inches wide, and weigh about 4 pounds. Twelve or 14 of each are used for this size of machine.

These series plates have the coils or wires enameled between ribs for increasing the adhesion of the enamel to the plate. It has been found by experience that 50 per cent. more current may be passed through a wire when placed between the ribs in this manner, without breaking the enamel, than where the wire is simply enameled on a plain surface. It is also a fact that these plates used in this way, will endure splashing of water about them without breaking the enamel, when the temperature is as high as will be reached in normal use.

Mr. Upton:-Mr. President, I would like to add one word, that I believe that at the Exposition at the Crystal Palace in London the invention of Mr. Carpenter's is one of the leading features That is, it is most talked about and most noticed by far. There is a large exhibition there, and it is meeting with a great deal of success.

[Adjourned to June 8th.] 